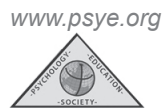

\title{
Variables Proximales relacionados con Violencia Escolar y Bullying en alumnado de bachillerato
}

\author{
Brenda Mendoza González*, Tania Morales Reynoso y Yazmín Arriaga Gómez \\ Facultad de Ciencias de la Conducta Universidad Autónoma del Estado de México
}

(Recibido 16 Mayo, 2015; Aceptado 18 Julio, 2015)

RESUMEN: La descripción del bullying se ha centrado principalmente en alumnado de educación básica, por lo que se hace necesario describir más este comportamiento en alumnado de educación media superior permitiendo así delinear protocolos de actuación y programas de intervención. El objetivo es conocer diferencias significativas entre grupos de alumnos que participan en violencia escolar, bullying y los que no se Involucran, en función de variables proximales como el comportamiento disruptivo, conflicto entre profesor-alumno, comportamiento antisocial y prosocial así como los efectos de la agresión en el contexto escolar. En el presente estudio participaron 1263 estudiantes de bachiller (rango de edad 15 a 20 años), 55\% mujeres y $45 \%$ varones (población total de una escuela preparatoria), los alumnos contestaron un instrumento en línea que permite identificar el tipo de agresión que ejerce o recibe, comportamiento antisocial, prosocial, así como los efectos del bullying. Los resultados permiten identificar que los alumnos se clasifican en tres tipos: No Involucrados, víctima acosador en violencia escolar y víctima/acosador de bullying. Se concluye que son los alumnos que participan en bullying los que exhiben comportamiento antisocial, tienen menos amigos, presentan más conductas disruptivas en el aula, y tienen más conflictos en la interacción con el profesorado.

Palabras clave: bullying, bachillerato, conducta antisocial, conducta pro social.

\section{Proximal Variables Related to School Violence and Bullying in High School Students.}

ABSTRACT: Description of bullying has mainly focused on pupils in basic education, for which it is necessary to describe more this behavior in students of education average superior to allowing delineate protocols of action and intervention programs. The objective is to know differences between groups of students participating in school violence, bullying and which not is involved, depending on variables proximal as disruptive behaviour, conflict between professors and students, antisocial and prosocial behavior as well as the effects of the aggression in the school context. In the present study participated 1263 Bachelor (age $15-20$ year range), $55 \%$ female and $45 \%$ male (total population of a school), the students answered a tool online that allows you to identify the type of aggression that exercises or receives antisocial, prosocial behavior, as well as the effects of bullying. The results allow to identify which students are classified into three 
types: not involved, harassing victim in school violence and bullying victim/stalker. It is concluded that they are students involved in bullying those who exhibit antisocial behavior, have fewer friends, present more disruptive behaviour in the classroom, and have more conflicts in the interaction with the faculty.

Keywords: bullying, high school, antisocial behaviour, conduct prosocial

\section{INTRODUCCIÓN}

A lo largo de las investigaciones desarrolladas en el mundo se han realizado contribuciones importantes en el estudio del comportamiento agresivo. Patterson (1982) ha realizado contribuciones para explicar a través de la teoría de la coerción como el comportamiento agresivo escala en los intercambios conductuales de una diada hasta llegar al abuso físico, Wilson y Herrnstein (1985), explican el desarrollo y mantenimiento del comportamiento agresivo a través de factores distales como la economía de un País, la competencia de la policía, el contexto familiar, la disponibilidad de sustancias nocivas para la salud, entre otras, Bandura (1973) ha definido el comportamiento agresivo como acciones negativas con o sin la intención de hacer daño a otra persona, explicando el desarrollo en los niños a través del aprendizaje de personas que modelan el comportamiento agresivo.

Se ha clasificado el comportamiento agresivo (Berkowitz , 1999; Dodge y Coie, 1987) para un mejor estudio, por lo que se ha descrito y se ha generado conocimiento que permite explicarlo (Bandura, 1973; Berkowitz,1999) y contribuir así, al desarrollo de programas de intervención (Kazdin, 2012).

Dodge y Coie (1987), lo clasifican el comportamiento agresivo en reactivo (reacción defensiva ante estímulos percibidos por el organismo como amenazantes) o proactivo o instrumental (se emplea la agresión para obtener un estímulo con alto valor para quien la emplea). Berkowitz (1999) hace una clasificación muy similar a la que hace Dodge y Coie (1987), distinguiendo a la agresión instrumental como (usarla para recibir un reforzamiento externo) y la agresión hostil (usada por la persona como una reacción al pánico que le genera el estímulo amenazante).

Olweus (1993), por su parte ha descrito el comportamiento agresivo exhibido en el contexto escolar, clasificado en dos subtipos: la violencia escolar y el bullying, empíricamente Pellegrini, Bartini y Brooks (1999), han demostrado que el alumnado con perfil de bully es quien usa el comportamiento agresivo definido como proactivo en episodios de bullying.

El tipo de agresión en la que se centra este estudio es el que se exhibe en los centros educativos, la violencia escolar y el bullying. Existe una clara diferencia entre ambos, el bullying se caracteriza por ser un comportamiento agresivo que tiene la intención de provocar daño a un alumno específico (se elige como víctima), se repite en el tiempo y existe desequilibrio de poder o fuerza entre sus protagonistas (Olweus, 1993), lo que no ocurre en violencia escolar que son episodios de agresión, sin embargo, no se dirige hacia un alumno en específico, 
no existe desequilibrio de poder entre sus protagonistas, y no se exhibe frecuentemente como el comportamiento de bullying, la diferenciación entre violencia escolar y bullying se hace necesario ya que de ello dependerá las estrategias para atenderlo y erradicarlo, si se confunde a ambos comportamientos se puede fortalecer aún más el comportamiento agresivo del alumnado con perfil de bully (Mendoza, 2014).

En más de cuarenta años de estudios internacionales se ha logrado describir el comportamiento de bullying, por ejemplo al inicio de su estudio se identificaban a tres participantes: el alumno víctima pura, el alumno bully y el alumno observador, actualmente se ha identificado a otros roles como el víctima/bully, rol que actualmente ha sido definido por muchos investigadores (Aguilera, Pedroza y Cervantes, 2013; Méndez y Cerezo, 2010; Mendoza, 2011; Rey del, Elipe y Ortega-Ruiz, 2012; Scholte et al., 2009; Yoneyama y Rigby, 2006).

La investigación del comportamiento de bullying ha permitido describirlo a partir de los estudios que tradicionalmente se han desarrollado desde los años 70's en Europa (Ortega, 2010), centrados básicamente en alumnado de primaria y secundaria. Recientemente se han iniciado esfuerzos por ampliar su estudio en el nivel medio superior, identificándose que es errónea la creencia de que el bullying únicamente ocurre en el nivel de educación básica (Brunstein et al., 2011).

Los estudios de bullying en bachillerato son escasos algunos se han centrado en describir la participación del alumnado en función de la frecuencia con la que lastiman a otros compañeros (Miranda, Serrano, Morales, Montes de Oca y Reynoso, 2013), generalmente para conocer la relación del bullying con otras variables de estudio, primero se identifican los roles de los alumnos participantes y posteriormente se identifica la relación con las variables de estudio, a partir de la tipología (Sevda y Sevim, 2012), ejemplo de ello, son los estudios desarrollados por Brunstein et al. (2013), que permiten determinar que el alumnado víctima se encuentra en mayor riesgo de exhibir depresión e incluso suicidio, en otros como el desarrollado por Magklara et al. (2012) se ha explorado la relación con variables socioeconómicas, determinándose que no hay conexión entre bullying y el nivel educativo de los padres, sin embargo, si se identificó que las familias con nivel socioeconómico bajo y en las que el padre no tiene ingreso socioeconómico, sitúan en mayor riesgo al alumnado de bachiller de participar como bully, para el alumnado con doble rol, se identifica que cuando las madres son pensionadas o no tienen ninguna actividad económica los sitúa en mayor riesgo de participar en episodios de bullying, en otros estudios con alumnos de bachillerato se ha identificado la relación de bullying tradicional y el cyberbullying (Kumazaki, Suzuki, Katsura, Sakamoto y Kashibuchi, 2011; Rey del, Elipe, y Ortega-Ruiz, 2012;).

En México los estudios en bachillerato, son escasos, a pesar de que desde la agenda de Gobierno se dicta su estudio y atención (Plan Nacional de Desarrollo [PND], 2013) fortaleciendo la necesidad de su atención a través de herramientas legales como la Ley General de los Derechos de las Niñas, Niños y Adolescentes (2014), en la que incluso se especifican sanciones para funcionarios que eviten 
ayudar a los niños involucrados en episodios de bullying, otro instrumento legal es la Ley General de Educación (2015) que en sus artículos 49 y 33, se establece la capacitación a profesorado y padres de familia respectivamente, para mejorar desde su función la convivencia en las escuelas, de hecho se tiene evidencia empírica de programas de intervención para disminuir el bullying desde el contexto familiar en educación básica (Mendoza, Pedroza y Martínez, 2014).

Para disminuir el comportamiento agresivo en el contexto escolar en nivel bachillerato, se hace necesario tener información precisa sobre la diferencia entre los grupos de alumnos que si participan en episodios de violencia escolar y bullying y los que no participan, permitiendo tener mayor información para construir programas para mejorar la convivencia en educación media superior, por lo que la presente investigación tiene como objetivo conocer diferencias significativas entre grupos de alumnos que participan en violencia escolar, bullying y los que no se Involucran, en función de variables proximales como el comportamiento disruptivo, conflicto entre profesor-alumno, comportamiento antisocial y prosocial así como los efectos de la agresión en el contexto escolar. Se establece como hipótesis que habrá diferencia significativa entre los grupos de alumnos identificados con respecto a las variables proximales del estudio.

\section{MÉTODO}

\section{Sujetos}

Participaron 1263 estudiantes de bachillerato, con rango de edad de 15 a 20 años (media 16.46, $\sigma=1.06)$, de ellos 697 son mujeres $(55 \%$ del total de alumnos participantes) y 566 son hombres ( $45 \%$ del total de alumnos participantes).

En el estudio participó el total de la población estudiantil inscrita, de una escuela preparatoria de la zona Centro del Estado de México (la única preparatoria de la zona, que pertenece a la Universidad Pública de dicho estado).

\section{Instrumento de medición}

El instrumento que se utilizó fue El Cuestionario de Medición de la Agresión Escolar e Intimidación en Bachillerato (Miranda, 2012). El instrumento se integra de 126 reactivos, con escala Likert de cinco opciones (no ha sucedido; dos o tres veces al mes; una vez a la semana; dos o tres veces a la semana; todos los días), lo que permite identificar si el episodio de agresión es violencia escolar (dos o tres veces al mes) o bullying (una vez a la semana, dos o tres veces a la semana o cotidianamente).

El Cuestionario se estructura en los siguientes factores: Tipo de agresión (recibida, ejercida u observada); Conducta prosocial iniciada y recibida; conducta antisocial (vandalismo; adicciones; consumo de alcohol y nicotina); Conducta Disruptiva; Conflicto con el profesorado (agresión que se ejerce hacia el profesor y agresión que se recibe del profesorado); Efectos del Bullying (Miedo, rendi- 
miento escolar, auto-percepción de su sentir por los conflictos en la escuela, alteraciones fisiológicas). El instrumento tiene un índice de confiabilidad de $=.95$.

Los Factores que lo componen y algunos ejemplos de los reactivos que los conforman se describen a continuación:

Tipo de agresión recibida, a) Víctima de Agresión Extrema (me han aventado objetos; me han golpeado; me han besado a la fuerza; me incomoda la forma en como tocan mi cuerpo), b) Víctima Exclusión (piden a otros compañeros que no se junten contigo; te dejan fuera de trabajo en equipos; han contado chismes de mí se burlan de mi) c) Víctima de Agresión de Gravedad media y cyberbullying (me han roto mis útiles escolares; escriben comentarios ofensivos sobre mí en las paredes, o bancas; escriben sobre mí en las redes sociales dañando mi imagen; me envían mensajes por internet para molestarme)

Tipo de agresión ejercida, a) Agresor extrema y ciber-bullying (he golpeado a un compañero para hacer algo que no quería; he obligado con amenazas a un compañero para que haga algo que no quiere; he acariciado a otros sin que quisieran; he intentado besar a la fuerza; he roto útiles escolares; he intimidado escribiendo notas ofensivas; he atemorizado a un compañero con mensajes por celular; he atemorizado a algún compañero enviándole mensajes por internet); b) Agresor de Exclusión y de gravedad medida (he pegado; le he aventado objetos a un compañero; me burlo de mis compañeros; he contado chismes de algún compañero; dejo fuera a compañeros en equipos; convenzo a mis compañeros para que no se junten con otro).

Conducta Pro-social iniciada (ayudo a mis compañeros cuando lo necesitan; guardo en secreto lo que me confían mis amigos; tengo amigos a quien invitar a mi casa; tengo amigos a quienes puedo confiar mis secretos; puedo ayudar a mejorar la relación con mis compañeros)

Conducta Pro-social recibida (los compañeros me prestan desinteresadamente algo que necesito; me defienden mis compañeros cuando alguien me pega o me amenaza; me eligen primero cuando se forman equipos; tengo amigos que me invitan a su casa)

Conducta antisocial a) Drogas (sé que mis compañeros han consumido algún tipo de droga; me han ofrecido algún tipo de droga; he consumido algún tipo de droga); b) Cigarro y alcohol (fumo: bebo alcohol, fumar o beber hace que me acepten mis compañeros); c) vandalismo (conozco compañeros que se juntan con alguna banda en la colonia donde vivo; he visto compañeros que toman pertenencias de otros, destruyen cosas de los demás, grafitean; pertenezco a una banda en la colonia en donde vivo).

Conducta Disruptiva (durante la clase he propiciado desorden; platico; interrumpo a mis compañeros; salgo constantemente del salón sin autorización)

Conflicto entres alumnado-profesorado a) Agresión del alumno hacia el profesor (aventar objetos; burlas; apodos; rumores maliciosos), b) Agresión del profesor hacia el alumno (se ha burlado de mi; se ha burlado de un compañero de clase; ha agredido a un compañero de clase; me ha agredido un maestro).

Efectos del bullying a) Miedo (he sentido miedo de ir a la escuela por el bu- 
llying que vivo; ya no me siento seguro en la escuela), b) Rendimiento Escolar (prefiero no ir a la escuela para evitar ver a algunos compañeros; he bajado de calificaciones porque no me siento a gusto) c) Auto percepción (me he sentido muy triste por la situación que vivo en la escuela; me siento desesperado por algunas cuestiones que vivo en la escuela) d) Alteraciones Psicológicas (he perdido el apetito a causa del acoso que vivo; me cuesta trabajo conciliar el sueño por las situaciones que vivo en la escuela; he perdido el interés de cuidar mi imagen cuando me presento en la escuela).

\section{Procedimiento}

La recolección de datos se hizo a partir de la aplicación del instrumento en línea (el servidor empleado fue el de la Universidad Autónoma del Estado de México), los responsables de la aplicación fue profesorado del plantel educativo por lo que fueron capacitados en el uso del instrumento de medida y en el servidor. Las autoridades escolares solicitaron autorización a los padres del alumnado participante. Se establecieron horarios para la aplicación del instrumento.

\section{Análisis de Datos}

La captura de los datos fue automática, la información se analizó en el programa estadístico SPSS (Versión 16).

Para identificar la clasificación del alumnado de acuerdo a su participación en episodios de violencia escolar y bullying, se desarrolló un análisis Multivariado, específicamente el análisis de conglomerados empleando del instrumento, el factor Tipo de agresión recibida y el factor Tipo de agresión ejercida.

Para conocer la existencia o no de diferencias significativas entre el grupo de alumnos que participan en: episodios de violencia escolar, episodios de bullying y los que no participan, en función del comportamiento disruptivo, conflicto entre profesor-alumno, comportamiento antisocial y prosocial se realizó un contraste de medias de dichas variables, a través análisis de varianza (ANOVA), teniendo como factor el conglomerado y como variable dependiente a cada una de las variables planteadas en el objetivo.

Para conocer la relación o no de las dos variables categóricas rol del alumnado y género, se realizó un análisis de $\chi 2$, se calcularon residuos corregidos para interpretar con precisión el significado de la asociación identificada.

\section{RESULTADOS}

Clasificación del alumnado en función de su participación en violencia o acoso escolar.

Para obtener la tipología, se realizó un análisis de clúster de $\mathrm{K}$ de medias combinando los reactivos de los factores del instrumento que miden la participación del alumnado como Agresor (ejercer agresión hacia sus compañeros) y 
Víctima (recibir agresión de sus compañeros) del instrumento ya descrito.

La situación adoptada con respecto a la tipología de situaciones en las que el alumnado participa en situaciones de violencia y acoso escolar, permite diferenciar dos grupos y uno más de un grupo de alumnos que no se involucran en situaciones de violencia escolar y tampoco de bullying, a continuación se describe cada uno:

1.- No involucrados: este grupo está formado por 1192 alumnos que no participan como víctimas o agresores en situaciones de violencia escolar y tampoco de bullying. De este grupo el $57 \%$ son mujeres (680 alumnas) y el $43 \%$ son hombres (512 alumnos).

Las alumnas de este grupo representan el $98 \%$ del total de mujeres participantes y los alumnos de este grupo representan el $90.5 \%$ del total de hombres participantes.

2.- Victima/Agresor en violencia Escolar: Este grupo está conformado por 67 alumnos los cuales reciben y ejercen agresión en la interacción con sus compañeros (agresión extrema, exclusión, gravedad media y ciberbullying). En este grupo el $19 \%$ son mujeres (13 alumnas) y el $81 \%$ son hombres (54 hombres). Las mujeres de este grupo representan el $2 \%$ del total de mujeres participantes y el $9.5 \%$ de total de los hombres participantes. El análisis de clúster los agrupa debido a que conforma la escala de Likert del instrumento respondieron que reciben y ejercen agresión "dos o tres veces al mes".

3.- Victima/Agresor en Acoso Escolar: este grupo está conformado por 5 alumnos los cuales por una parte reciben agresión extrema, exclusión, gravedad media y ciberbullying y a la vez la ejercen hacia otros alumnos. En este grupo el $81 \%$ son hombres y el $20 \%$ son mujeres. Las mujeres de este grupo representan $0.2 \%$ del total de las alumnas participantes y los hombres de este grupo representan el $0.6 \%$ del total de hombres participantes. El análisis de clúster agrupa a estos alumnos al identificar su participación al recibir o dirigir agresión a otros compañeros "una vez a la semana" o "todos los días" (de acuerdo a la escala de Likert).

En la figura 1, se muestran las medias de los conglomerados para cada una de las situaciones en las que el alumnado participa (descritas anteriormente). 


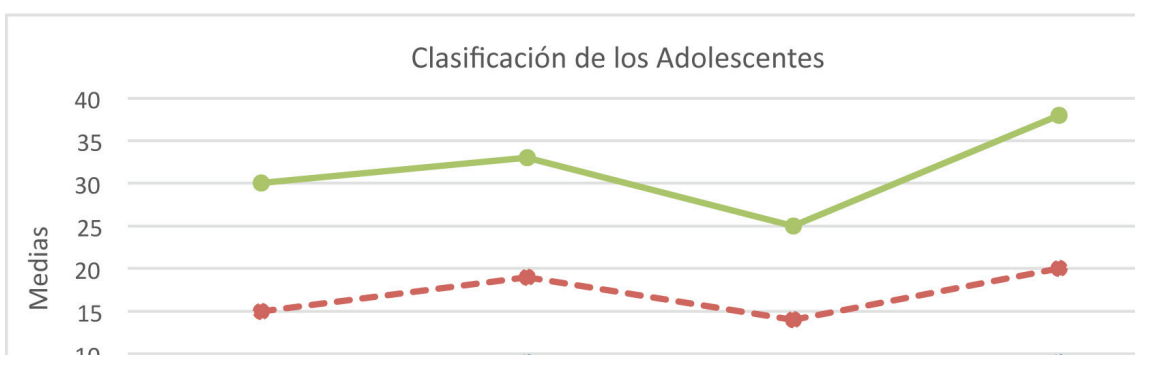

Figura 1.Puntaje de las medias obtenidas en los factores: Tipo de Agresión Ejercida y Tipo de Agresión Recibida del Instrumento aplicado al alumnado de bachillerato

\section{Género y rol de participación}

Se utilizó el estadístico $\chi^{2}$ para conocer la dependencia entre la variable género y la tipología de adolescente como víctima/agresor en la interacción con sus pares. Los resultados encontrados indican que las dos variables son dependientes, es decir, el participar en situaciones de violencia y acoso escolar con el papel de agresor/víctima se relaciona con ser hombre o mujer $\chi^{2}(2,1263)=32.172$, $\mathrm{p}<.001)$. Los resultados indican que las mujeres no participan en episodios de violencia y acoso escolar (residuo corregido con valor 5) y son los alumnos varones los que se encuentran en mayor riesgo de participar desempeñando el doble rol (víctima/agresor) en situaciones de violencia escolar (valor de residuo corregido 5.6).

Para conocer la diferencia entre los tres grupos identificados: víctima/agresor en violencia escolar, víctima/agresor en bullying, y No Involucrado, en función del comportamiento disruptivo en el aula, conflictividad en la relación con el profesorado; comportamiento antisocial y comportamiento prosocial) se desarrolló un contraste de medias a través del ANOVA, teniendo como factor el conglomerado y como variable dependiente cada una de las variables de contraste.

\section{Comportamiento Disruptivo en el aula}

El contraste de medias para la variable Comportamiento Disruptivo indica que hay diferencias significativas entre los tres grupos $\mathrm{F}$ ( 2 y 10.061. $)=96.540$, $\mathrm{p}<.001$. Las pruebas de contraste post hoc indican que el alumnado con doble rol (víctima/acosador) en bullying, presenta media más alta que $(\overline{\mathrm{X}}=16.60 ; \sigma=3.05)$ que el grupo de alumnos que No se Involucra $(\bar{x}=6.06 ; \sigma=2.45)$.

\section{Conflicto entre profesor-alumno}

Con respecto a la variable Agresión del alumno hacia el profesor, el contraste de medias permite identificar que existen diferencias significas entre los 
grupos de alumnos identificados, $\mathrm{F}(2$ y 10.013$)=102.625, \mathrm{p}<.05$. Las pruebas de contraste post hoc, señalan que el alumnado con doble rol víctima/acosador en bullying presenta media más alta (avientan bolitas de papel; se burlan de algún maestro cuando están en clase; le ponen apodos; dicen rumores maliciosos), $\overline{\mathrm{X}}=17 ; \sigma=3.08$, que el alumnado que No se Involucra $(\overline{\mathrm{X}}=5.24 . ; \sigma=1.83)$ en episodios de violencia escolar y bullying.

Con respecto a la variable Agresión del profesor hacia el alumno, existen también diferencias significativas entre el grupo de alumnos, $F(2$ y 10.011) $=$ (74.270), $\mathrm{p}<.05$ el alumnado Víctima/acosador en episodios de bullying, presenta media más alta $\overline{\mathrm{X}}=17 ; \sigma=3.74$, (recibe burlas del profesor; u otro tipo de agresiones), significativamente más que el alumnado que No se Involucra $(\overline{\mathrm{X}}=$ $5.21 ; \sigma=2.11)$.

\section{Comportamiento antisocial}

Con respecto a la variable Beber y fumar, el contraste de medias permite identificar diferencias significativas entre los grupos de alumnos $F(2$ y 10.006) $=$ $37.290, \mathrm{p}<.05$., las pruebas de contraste Post hoc permiten identificar que el alumnado con víctima/acosador en bullying tiene media significativamente más alta $(\overline{\mathrm{X}}=13.60 ; \sigma=1.34)$ que el alumnado identificado como No Involucrado $(\overline{\mathrm{X}}=9.38 ; \sigma=1.81)$,

Con respecto a la variable Conducta de consumo de drogas el contraste de medias permite identificar diferencias significativas entre los grupos de alumnos $\mathrm{F}(2$ y 10.008$)=56.835, \mathrm{p}<.05$, las pruebas de contraste Post hoc permiten identificar que el alumnado que participa como vtima/acosador en bullying manifiesta haber observado cuando una persona ofrece droga a otro y manifiesta consumir drogas ( $\overline{\mathrm{X}}=13 ; \sigma=2.82)$ significativamente más que el alumnado que No se Involucra en episodios de bullying ( $\bar{X}=4.02 ; \sigma=1.65)$.

Para la variable Actos vandálicos, el contraste de medias muestra diferencias significativas entre los grupos $\mathrm{F}(2$ y 10.040) $=(166.698), \mathrm{p}<.05$, las pruebas de post hoc, identifican que el víctima/acosador en bullying $(\overline{\mathrm{X}}=13.60 ; \sigma=1.34)$ manifestaron conocer a personas que forman parte de un grupo vandálico (i.e. "destruyen cosas de los demás"; "hacen grafiti ") significativamente más que el grupo de alumnos que No se Involucran en episodios de bullying $(\overline{\mathrm{X}}=3.58$; $\sigma=1.28)$,

\section{Conducta Pro social}

Con el objetivo de describir la diferencia de medias de los tres grupos de alumnos participantes en función de la variable Conducta pro social (factores del instrumento Conducta pro social iniciada y recibida), se realizó un análisis de varianza.

Con respecto a la variable Conducta Prosocial Iniciada se identificó que existe diferencia de medias en los tres grupos de alumnos $F(2$ y 10.08) $=20.723$, 
$\mathrm{p}<.001$. Las pruebas de contraste post hoc, permitieron identificar que el alumnado que No se Involucra en episodios de bullying ( $\overline{\mathrm{X}}=26.36 ; \sigma=3.20)$, "ayuda más a sus compañeros" y "guardan más secretos" significativamente más que el alumnado que se involucra en violencia escolar $(\bar{X}=24.00 ; \sigma=3.33)$.

Con respecto a la variable Conducta Prosocial Recibida F (2 y 10.06) $=$ $10.236, \mathrm{p}<.001$, las pruebas Post hoc permiten identificar que el alumnado clasificado como No Involucrado presenta media significativamente más alta $\overline{\mathrm{X}}=$ $16.21 ; \sigma=2.63$, que el alumnado que se involucra en violencia escolar $(\overline{\mathrm{X}}=$ $15.14 ; \sigma=2.40$ ).

\section{Efectos del acoso escolar}

Con respecto a la variable miedo se identificó diferencia entre los grupos $\mathrm{F}$ $(2$ y 10.015) $=97.479, \mathrm{p}<.001$, las pruebas Post hoc permiten identificar que el alumnado víctima/acosador en bullying tienen medias significativamente más alta $(\overline{\mathrm{X}}=12.80 ; \sigma=1.64)$ que el alumnado víctima de violencia escolar $(\overline{\mathrm{X}}=$ 9.11; $\sigma=1.67)$ y que el alumnado No Involucrado $(\bar{X}=1.19 ; \sigma=.62)$.

Con respecto a la variable Rendimiento escolar también se identificaron diferencias entre los grupos de alumnos $\mathrm{F}(2$ y 10.09$)=50.115$, $\mathrm{p}<.05$, las pruebas post hoc, permiten identificar que el alumnado víctima/acosador en bullying tiene medias significativamente más elevada $(\bar{X}=4.40 ; \sigma=.89)$ que el alumnado víctima/acosador de violencia escolar $(\overline{\mathrm{X}}=2.26 ; \sigma=1.21)$ y que el alumnado del grupo No Involucrados $(\overline{\mathrm{X}}=1.19 ; \sigma=0.62)$.

Con respecto a la variable Auto-percepción negativa se identifica diferencias significativas entre los grupos de alumnos $\mathrm{F}(2$ y 9.97) $=42.147, \mathrm{p}<.05$., las pruebas post hoc, permiten identificar que el alumnado víctima/acosador en bullying tienen media significativamente más alta $\overline{\mathrm{X}}=8.40 ; \sigma=2.07$ (tristeza y desesperación debido a la agresión en la escuela) más que el alumnado víctima/ acosador de violencia escolar $\bar{X}=4.04 ; \sigma=2.07$ y que el alumnado identificado como No Involucrados $(\overline{\mathrm{X}}=2.19 ; \sigma=0.66)$.

Con respecto a la variable Alteraciones Fisiológicas se identificaron diferencias significativas entre los grupos de alumnos $\mathrm{F}(2$ y 9.985$)=24.306, p<.05$, las pruebas Post hoc, señalan que el alumnado víctima/acosador en bullying tiene media significativamente más alta $\overline{\mathrm{X}}=15.20 ; \sigma=3.63$, (falta de apetito, dificultades para conciliar el sueño y pérdida de interés por su auto imagen) más que el alumnado No Involucrado $(\overline{\mathrm{X}}=9.21 ; \sigma=1.37)$

\section{DISCUSIÓN}

El objetivo de la presente investigación es conocer la existencia de diferencias significativas entre grupos de alumnos que participan en violencia escolar, bullying y los que no se Involucran, en función de variables proximales como comportamiento disruptivo, conflicto entre profesor-alumno, comportamiento antisocial y prosocial así como los efectos de la agresión en el contexto escolar, 
en estudiantes de nivel medio superior.

Para identificar los grupos de alumnos, se obtuvo la tipología del alumnado que participa o no en episodios de violencia y acoso escolar, identificándose que fueron tres tipos principalmente: 1) Alumnos que no participan en situaciones de agresión en el contexto escolar, por lo que se les determino No Involucrados; 2) alumnado que se involucra recibiendo y ejerciendo agresión en la interacción con sus compañeros con frecuencia dos veces por mes, a quienes se les identificó como alumnado con doble rol Víctima/acosador en episodios de violencia escolar y 3) el alumnado que participa ejerciendo y recibiendo agresión de todo tipo (exclusión de gravedad media y extrema) cotidianamente, a este grupo se le identificó como víctima/acosador en episodios de bullying, estos resultados son consistentes con investigaciones desarrolladas por otros autores quienes han identificado al alumnado que No se Involucra (Díaz-Aguado, Martínez, y Martín-Seoane, 2004; Mendoza, 2011) y al alumnado que desempeña doble rol víctima/acosador (Aguilera, Pedroza y Cervantes, 2013; Méndez y Cerezo, 2010; Mendoza, 2011; Rey del, Elipe, y Ortega-Ruiz, 2012; Scholte et al., 2009; Yoneyama y Rigby, 2006).

Los resultados permiten concluir que el alumnado que participa en episodios de bullying con doble rol víctima/acosador, son varones y se caracterizan por: exhibir mayor comportamiento disruptivo en el aula, mayor conflicto con el profesorado (ejercer y recibir maltrato en la interacción con el profesorado, por ejemplo avientan bolitas de papel; se burlan de algún maestro cuando están en clase; le ponen apodos; dicen rumores maliciosos), además exhiben comportamiento antisocial como participar en actos de vandalismo, tienen acceso a drogas, fuman y toman bebidas alcohólicas, esto último, reconocen que lo hacen como una forma de aceptación en su grupo de pares, estos resultados son congruentes con lo identificado con otros autores (Azevedo da Silva et al., 2012).

Los resultados de esta investigación fortalecen la evidencia empírica de Mendoza (2011), quien identifica una conexión entre el comportamiento de bullying con la agresión en la interacción profesor-alumno, identificando que la reciprocidad coercitiva entre alumnos que participan en bullying y el profesor, predice escalamiento del comportamiento agresivo en su relación.

Con respecto al comportamiento antisocial, los resultados de esta investigación también fortalecen a otras investigaciones en las que se ha determinado que los alumnos que "no entran" a clases, usan drogas y beben alcohol, son alumnos que participan en episodios de bullying con el doble rol (Azevedo da Silva et al., 2012).

En investigaciones específicas con alumnado de bachillerato que participa en episodios de bullying, se han identificado como un indicador del contexto familiar, es el uso de disciplina inconsistente, autoritaria con agresión (Sevda y Sevim, 2012), de hecho se ha identificado que los alumnos que participan en bullying y cuyos padres mantienen un bajo monitoreo y supervisión de sus hijos se predice el desarrollo de comportamiento antisocial (Vassallo, Edwards, Renda y Olsson, 2014), por lo que este estudio permite delinear pautas de prevención 
dirigidas también a Padres de Familia de alumnos de bachillerato, prácticas de crianza positivas, consistentes que no dependen del estado de humor del padre (Mendoza et al., 2014)que les permita fortalecer la comunicación y confianza entre padres e hijos, ejerciendo disciplina sin hacer uso de la coerción.

Con respecto al comportamiento antisocial, algunas investigaciones han determinado que el alumnado que se encuentra en mayor riesgo de desarrollar este tipo de comportamiento se debe a diversos factores individuales como impulsividad, exhibición de enojo con agresión, déficits para resolver conflictos, por lo que será necesario también como parte de un programa integral de protección y atención dirigido al alumnado de bachiller, técnicas de auto control que el propio alumnado pueda emplear para prevenir involucrarse en episodios de agresión entre pares.

Los factores que fueron identificados en el alumnado que No se Involucra en episodios de bullying fueron: llevarse bien con el profesorado; no exhibir comportamiento disruptivo en clase; no exhibir comportamiento antisocial (acceso a drogas, beber alcohol, fumar nicotina y no ser parte de grupo vandálico). Con respecto a la relación que establecen con sus compañeros, se caracterizan por exhibir comportamiento pro-social hacia otros compañeros como ayudar (cuando identifican que alguien lo necesita), en la relación con sus amigos se caracteriza por tener amigos que confían en él (guardan secretos), tiene amigos a quien invitar a su casa y en general ayudan para mantener y mejorar una buena relación con sus amigos y compañeros de clase.

Siguiendo con la descripción de los alumnos No Involucrados, se identifica que reciben comportamiento pro social de sus compañeros ya que a estos alumnos les prestan desinteresadamente algo cuando lo necesitan; les "defienden" cuando alguien les pega o les amenaza, cuando se forman equipos se identificó que le eligen en primer lugar cuando se forman equipos; en la relación con sus amigos, reciben invitaciones para ir a la casa de sus amigos.

Estos resultados son congruentes con lo reportado por Oliveira de, Lisboa, Koller y Sousa de, (2011) quienes identificaron que los alumnos que no participan en bullying exhiben comportamiento pro social y son populares entre sus compañeros de clase, en contraparte Uribe, Orcasita y Aguillón (2012) identificaron que los alumnos que participan en bullying carecen de red de apoyo (amigos).

Con respecto a los efectos del acoso escolar se identificó que son los alumnos con doble rol víctima/acosador los que exhiben más efectos negativos como resultado de los episodios de bullying como: dificultad para dormir, pérdida de apetito; pérdida de interés por cuidar su imagen; sienten tristeza y desesperación, lo que es consistente con lo reportado con otras investigaciones (Magklara et al., 2012) en la que se identificó que el alumnado con doble rol se encuentran en mayor riesgo de presentar ansiedad, depresión, dificultad para dormir, preocupación, fatiga, cambios constantes de humor. Dichas evidencias fortalecen la urgente necesidad de construir protocolos de actuación, ya que es indispensable dar atención psicológica al alumnado que participa en bullying como víctimas 
o acosadores, lo anterior se hace necesario debido a que en escuelas Mexicanas no existe algún protocolo de actuación en operación que guie a los docentes para que deriven a alguna institución de salud de forma sistemática y consistente cada vez que identifiquen a alumnos que se involucran en episodios de bullying.

Derivado de las variables (comportamiento disruptivo, conflicto entre profesor-alumno, comportamiento antisocial y prosocial, así como los efectos de la agresión en el contexto escolar) que se relacionan con la participación o no del alumnado en episodios de bullying es posible contar con indicadores que guiarán el desarrollo de programas para prevenir y/o atender episodios de bullying en el nivel medio superior, estableciéndose como los principales componentes del programa: estrategias en habilidades sociales, que les permitan establecer y mantener redes de amigos que se caractericen por exhibir y recibir comportamiento pro social (ayudar, trabajar en equipo, comunicarse asertivamente, cooperar y mostrar confidencialidad entre pares) que permita al alumnado fortalecer su red social (incluso en actividades de ocio), así como la calidad de las relación de amistad (caracterizados por exhibir comportamiento de ayuda y confidencialidad entre pares); estrategias de auto control, que permitan al alumnado comunicar lo que opinan o sienten sin lastimar a otros (incluyendo autoridades escolares como el Profesorado); estrategias dirigidas a la prevención de adicciones y comportamiento antisocial.

Una de las limitantes del presente estudio fue no obtener información más amplia de algunas variables de medida como la del comportamiento anti social, de adicciones y de la reciprocidad coercitiva entre profesorado-alumnado, por lo que se sugiere en futuros estudios medir las variables con instrumentos específicos.

\section{REFERENCIAS}

Aguilera,S. J., Pedroza, F. J. y Cervantes, A.R. (2013). Roles desempeñados en el Bullying: implicaciones prácticas. En S. J. Aguilera y F. J. Pedroza (Eds). $L a$ construcción de identidades agresoras: el acoso escolar en México (31-48). México: CONACULTA. Azevedo da Silva, R., De Azevedo, T., Jansen, K., Dias-de-Mattos, L., Vanila, R. Sica, A.L.,

Lessa, B., and Tavares, R. (2012). Bullying and Associated Factors in

Adolescents aged 11 to 15 years. Trends in Psychiatry and Psychotherapy, 34(1), 19-24.

Bandura, A. (1973). Aggression: A social learning analysis. Englewood Cliffs, NJ: Prentince Hall.

Berkowitz, L. (1999). Anger. In T. Dalgleish y M. Power (Eds). Handbook of Cognition and Emotion (pp 411-428). Chichester, UK: Wiley.

Brunstein, A., Kleinman, M., Altschuler, E., Marroco, F., Amakawa, L., and Gould, M. (2011). High school bullying as a Risk for later depression and suicidality. Suicide life threat Behavior, 41(5), 501-516.

Brunstein, A., Kleinman, M., Altschuler, E., Marroco, F., Amakawa, L., and Gould, M. (2013). Suicidal Adolescents' Experience with bullying perpetra- 
tion and victimization during high school as risk factors for later depression and suicidality. Journal of Adolescent Health, 53, 537-542.

Díaz-Aguado, M.J., Martínez, R., y Martín-Seoane, G. (2004). Prevención de la Violencia y lucha contra la exclusión desde la adolescencia: la violencia entre iguales en la escuela y el ocio. Volumen 2. Programa de intervención y estudio experimental. Madrid: INJUVE.

Dodge, K.A. and Coie, J.D. (1987) Social-information-processing factors in reactive and proactive aggression in children's peer groups. Journal of Personality Social Psychology,53,1146-1158.

Kazdin, A. (2012). Behavior Modification in Applied Setting. USA: Waveland, Press Inc.

Kumazaki, A., Suzuki,K., Katsura, R., Sakamoto, A., and Kashibuchi, M. (2011) The Effects of Netiquette and ICT Skills on School- bullying And cyberbullying: The two-wave Panel Study of Japanese elementary, secondary and high school students. Social and behavioral Sciences, 29,735-741. Recuperado de www.sciencedirect.com/.../pii/S1877042811027601

Ley General de los Derechos de las Niñas, niños y adolescents (2014). Diario Oficial de la Federación (Publicado 4 de Diciembre del 2014). Recuperado de https://www.sep.gob.mx/.../ley_general_ninasninosyadolescentes.

Ley General de Educación (2015). Diario Oficial de la Federación (Publicado el 20 de abril del 2015) recuperado de www.diputados.gob.mx/LeyesBiblio/ pdf/137_200415.pdf

Magklara, K., Skapinakis, P., Gkatsa, T., Bellos, S., Araya, R., Sylianidis, S., and Mavreas, V. (2012). Bullying behavior in schools, socioeconomic position and psychiatric

morbidity: a cross-sectional study in late adolescents in Greece. Child and Adolescent Psichiatry and Mental Health, 6(8), 2-13.

Méndez, I. y Cerezo, F. (2010) Bullying y factores de riesgo para la salud en estudiantes de Secundaria. European Journal of Education and Psychology, 3(2), 209-218.

Mendoza, B. (2011). Bullying entre pares y el escalamiento de agresión en la relación profesor-alumno. Psicología Iberoamericana, 19(1), 58-71.

Mendoza, B. (2014). Los Múltiples Rostros del Acoso escolar ( ed.). México: Pax-México.

Mendoza, B., Pedroza, F. J. y Martínez, K. (2014). Prácticas de Crianza Positivas: Entrenamiento a Padres para reducir bullying. Acta de Investigación Psicológica, 4(3), 1793-1808.

Miranda, D. (2012). Validación del Cuestionario de medición de la agresión escolar y acoso en bachillerato. Facultad de Ciencias de la Conducta UAEM. Documento no publicado

Miranda, D., Serrano, M. J., Morales, T., Montes de Oca, J. Z. y Reynoso, B. (2013). Agresión y acoso en el nivel medio superior: El caso de la Universidad Autónoma del Estado de México. En S. J. Aguilera y F. J. Pedroza (Eds.). La construcción de identidades agresoras: el acoso escolar en México. Mé- 
xico: CONACULTA

Oliveira de T., Lisboa, C., Koller, S., and Sousa de, D. (2011). Aggression and Pro-Sociability: Risk and Protective Dynamics in Popularity and Bullying Processes Psykhe, 20(2), 53-63

Ortega, R. (2010). Agresividad injustificada, bullying y violencia escolar. Madrid: Alianza Editorial.

Patterson, G. R.(1982). Coercive Family Process, Eugene, O.R: Castalia.

Pellegrini, A., Bartini, M. y Brooks, F. (1999). School bullies, victims and aggressive victims. Journal of Educational Psychology, 91, 216-224

Plan Nacional de Desarrollo ([PND]2013). Plan Nacional de Desarrollo 20132018. Diario Oficial de la Federación. Secretaría de Gobernación. [En línea]. Disponible en: http://www.dof.gob.mx/nota_detalle.php?codigo=5299465\& fecha $=20 / 05 / 2013$.

Rey del, R. Elipe, P. y Ortega-Ruiz, R. (2012). Bullying and cyberbullying: Overlapping and predictive value of the co-occurrence. Psicothema, 24(4), 608-613.

Scholte, R.H., Overbeek, G., Brink, G., Rommes, E., de Kemp, R., Goossens, L. and Engels, R. (2009). The significance of reciprocal and unilateral frienships for peer victimization in adolescence, Journal Youth Adolescence, 38, 89-100.

Sevda, A. and Sevim, S. (2012). Effect Of High School Students' Selfconcept and Family Relationships on peer bullying. Revista Brasileira em Promoção da Saúde, 25 (4), 405-412.

Yoneyama, S. and Rigby, K. (2006). Bullying/Victim student and classroom climate. Youth Studies Australia, 25(3), 34-41

Uribe, A. F., Orcasita, L., y Aguillón-Gómez, E. (2012). Bullying, redes de apoyo social y funcionamiento familiar en adolescentes de una institución educativa de Santander, Colombia Psychologia. Avances de la disciplina European Journal of Education and Psychology, 3 (2), 209-218.

Vassallo, S., Edwards, B., Renda J., and Olsson, C. (2014). Bullying and Antisocial Behavior: Protective Factors. Journal of school violence, 13, 100-124

Wilson, J. and Herrnstein, R.(1985) Crime and Human Nature. New York: Simon and Schuster, Inc. 